\title{
MODERN HYDRODYNAMICAL THEORY, WITH SPECIAL REFERENCE TO AERONAUTICS*
}

\author{
BY F. D. MURNAGHAN
}

\section{A. General Notions and Theorems}

1. Particle and Local Differentiation. In hydrodynamical theory matter is regarded as distributed continuously, but its various points are supposed to be capable of identification; we shall refer to them as particles. In order to identify a particle, a material reference frame is introduced, and we shall suppose for simplicity that this is a rectangular Cartesian frame. At any convenient initial instant $t=t_{0}$, the coordinates of a particle may be denoted by $(a, b, c)$; and at any later instant the coordinates of the same particle may be denoted by $(x, y, z)$. We make the assumption that $(x, y, z)$ are differentiable functions of $a, b, c$, and $t$. This assumption

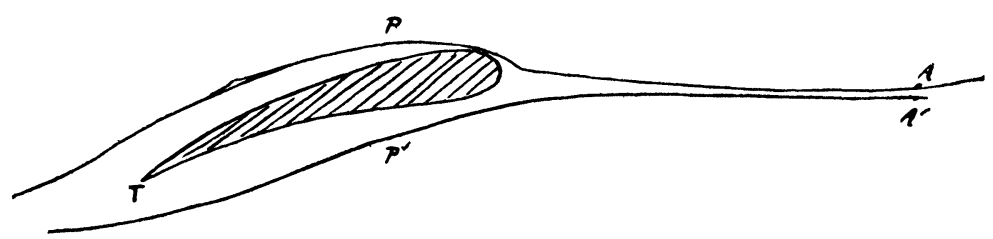

FIG. 1

must be carefully noted since the principal object of interest to us later on will be the flow of a fluid around an obstacle and the fluid will divide at the obstacle. Thus two particles initially adjacent (as $A$ and $A^{\prime}$ in Fig. 1) will not be adjacent when separated by the obstacle as at $P$ and $P^{\prime}$. Our subse-

* This paper is a somewhat formal and detailed presentation of the contents of an address delivered at the invitation of the program committee at the meeting of this Society on October 29, 1927. The adjective modern in the title of the address refers to the first quarter of the present century, but in order to make the paper easily intelligible to mathematical readers who have not specialized in hydrodynamics, I give a statement of the more important results which had been previously obtained. 
quent remarks will apply, then, separately to the two portions of the fluid which separate at the obstacle but not to the fluid as a whole. A particle may be identified either by means of $(a, b, c, t)$ which are known as the particle, or Lagrangian, variables or by means of $(x, y, z, t)$ which are known as the local, or Eulerian, variables; we shall denote time differentiation when the particle variables are used by $d / d t$ and when the local variables are used by $\partial / \partial t$. The velocity components $(d x / d t, d y / d t, d z / d t)$ being denoted by $(u, v, w)$, we have the relation

$$
\frac{d}{d t}=\frac{\partial}{\partial t}+u \frac{\partial}{\partial x}+v \frac{\partial}{\partial y}+w \frac{\partial}{\partial z} .
$$

In particular the acceleration components $(d u / d t, d v / d t$, $d w / d t)$ are given, in terms of the local variables, by formulas of the type

$$
\frac{d u}{d t}=\frac{\partial u}{\partial t}+u \frac{\partial u}{\partial x}+v \frac{\partial u}{\partial y}+w \frac{\partial u}{\partial z}
$$

The paths of the various particles are found, if $(u, v, w)$ are known in terms of the local variables, by integrating the three equations

$$
\frac{d x}{u}=\frac{d y}{v}=\frac{d z}{w}=d t .
$$

At any given instant $t=t_{1}$ the stream-lines are given by integrating the two equations

$$
\frac{\delta x}{u_{1}}=\frac{\delta y}{v_{1}}=\frac{\delta z}{w_{1}},
$$

where $u_{1}=u\left(x, y, z, t_{1}\right)$, and so on. The $\infty^{3}$ paths must then be distinguished from the $\infty^{2}$ stream-lines. They coincide geometrically when the stream-lines are permanent, that is, when $\partial u / \partial t=0, \partial v / \partial t=0, \partial w / \partial t=0$ identically in $t$. In this case the motion is said to be steady, and in the applications with which we shall be concerned steady motion is assumed. 
2. The Principle of Conservation of Mass. This postulates the existence of a positive density function $\rho$, which we assume to be differentiable, and such that the volume integral $\int \rho \delta(x, y, z)$ does not vary with $t$, the volume of integration consisting of the same particles. In other words $\int \rho \delta(x, y, z)$ is an integral invariant of the equations (3). If the initial value of $\rho$ be denoted by $\rho_{0}$, the differential form of statement of the principle of conservation is, in terms of the particle variables,

$$
\rho \frac{d(x, y, z)}{d(a, b, c)}=\rho_{0},
$$

and in terms of the local variables,

$$
\frac{\partial \rho}{\partial t}+\frac{\partial}{\partial x}(\rho u)+\frac{\partial}{\partial y}(\rho v)+\frac{\partial}{\partial z}(\rho w)=0 .
$$

This equation is known as the equation of continuity and it may be put in the equivalent form

$$
\frac{d \rho}{d t}+\rho\left(\frac{\partial u}{\partial x}+\frac{\partial v}{\partial y}+\frac{\partial w}{\partial z}\right)=0 .
$$

For an incompressible fluid $d \rho / d t=0$ and the equation of continuity is

$$
\frac{\partial u}{\partial x}+\frac{\partial v}{\partial y}+\frac{\partial w}{\partial z}=0 .
$$

3. The Circulation Integral. With any closed curve $C$ of particles we may associate, at any instant $t$, the number

$$
\Gamma=\oint(u \delta x+v \delta y+w \delta z),
$$

where $\delta$ indicates the differential with respect to the parameter of the curve. The integral $\Gamma$ is known as the circulation around the curve; and an application of Stokes' theorem enables us to write it in the equivalent form 


$$
\Gamma=\int \xi \delta(y, z)+\eta \delta(z, x)+\zeta \delta(x, y)
$$

where

$$
\xi=\frac{\partial w}{\partial y}-\frac{\partial v}{\partial z}, \quad \eta=\frac{\partial u}{\partial z}-\frac{\partial w}{\partial x}, \quad \zeta=\frac{\partial v}{\partial x}-\frac{\partial u}{\partial y},
$$

are the vorticity components and the surface of integration is bounded by $C$. A simple calculation shows that

$$
\begin{gathered}
\frac{d \Gamma}{d t}=\oint\left\{\left(\frac{\partial}{\partial y} \frac{d w}{d t}-\frac{\partial}{\partial z} \frac{d v}{d t}\right) \delta(y, z)\right. \\
\left.+\left(\frac{\partial}{\partial z} \frac{d u}{d t}-\frac{\partial}{\partial x} \frac{d w}{d t}\right) \delta(z, x)+\left(\frac{\partial}{\partial x} \frac{d v}{d t}-\frac{\partial}{\partial y} \frac{d u}{d t}\right) \delta(x, y)\right\} .
\end{gathered}
$$

Hence the necessary and sufficient conditions that $d \Gamma / d t=0$ for every closed curve $C$ are

$$
\frac{\partial}{\partial y} \frac{d w}{d t}-\frac{\partial}{\partial z} \frac{d v}{d t}=0
$$

and so forth. In other words, $\Gamma$ is a (relative) integral invariant of the equations (3) provided that the acceleration components are derivable from a potential function $Q$, that is, provided that

$$
\frac{d u}{d t}=-\frac{\partial Q}{\partial x}, \quad \frac{d v}{d t}=-\frac{\partial Q}{\partial y}, \quad \frac{d w}{d t}=-\frac{\partial Q}{\partial z},
$$

and conversely. In our applications this will be the situation. A particular instance of importance occurs when $\Gamma=0$ throughout a region of the fluid; then the vorticity components $(\xi, \eta, \zeta)$ are zero and the motion is said to be irrotational in that region. The velocity components are derivable from a single function $\phi$ as follows :

$$
u=\frac{\partial \phi}{\partial x}, \quad v=\frac{\partial \phi}{\partial y}, \quad w=\frac{\partial \phi}{\partial z} ;
$$

and $\phi$ is called the velocity potential. 
4. The Fundamental Equations and the Concept of a Perfact Fluid. If we focus our attention on a particular point $(x, y, z)$ of the fluid, the orientation of a particular element of area $\delta S$ through $(x, y, z)$ may be specified by means of the direction cosines $(l, m, n)$ of one of the two directed normals to $\delta S$ and we may call the side of $\delta S$ towards which the normal is drawn its positive side. Then the fluid on the negative side of $\delta S$ exerts on the fluid on the positive side a force whose components may be denoted by $\left(P_{x} \delta S, P_{y} \delta S, P_{z} \delta S\right)$, where $\left(P_{x}, P_{y}, P_{z}\right)$ are given in terms of $(l, m, n)$ and nine coefficients $\left(p_{x x}, p_{x y}, \cdots, p_{z z}\right)$ by the equations

$$
\begin{aligned}
& P_{x}=l p_{x x}+m p_{y x}+n p_{z x}, \\
& P_{y}=l p_{x y}+m p_{y y}+n p_{z y}, \\
& P_{z}=l p_{x z}+m p_{y z}+n p_{z z} .
\end{aligned}
$$

The nine coefficients $\left(p_{x x}, p_{x y}, \cdots, p_{z z}\right)$ constitute what is called the stress or pressure tensor. If the fluid is subjected to a system of forces $(X, Y, Z)$ per unit mass, a simple application of the fact that the total force acting on any portion of the fluid is equal to the time rate of change of its linear momentum yields the equations

$$
\begin{array}{cl}
\rho \frac{d u}{d t}=\rho X-\left(\frac{\partial p_{x x}}{\partial x}+\frac{\partial p_{y x}}{\partial y}+\frac{\partial p_{z x}}{\partial z}\right) ; \\
\rho \frac{d v}{d t}=\quad ; \rho \frac{d w}{d t}=
\end{array}
$$

In deriving these we have used the fact that if there are surface forces, whose components are $(F, G, H)$ per unit area, acting on the fluid then (see (1)) we have

(14) $F+l p_{x x}+m p_{y x}+n p_{z z}=0 ; G+=0 ; H+=0$;

where $(l, m, n)$ are the direction cosines of the outward drawn normal. The theorem that the moment of the forces acting on the fluid about any axis is equal to the time rate of change of its angular momentum about this axis yields the fact that the pressure tensor is symmetric, that is, 


$$
p_{z y}=p_{y z}, \quad p_{x z}=p_{z x}, \quad p_{y x}=p_{x y} .
$$

A perfect fluid is by definition one such that the stress across any element is normal to it; it is this quality of inability to withstand tangential stresses that distinguishes fluids from solids. The equations (12) combined with $P_{x}: P_{y}: P_{z}$ $=l: m: n$ for arbitrary values of the ratios $l: m: n$ show that the pressure tensor for a perfect fluid is the scalar tensor $(p, 0,0 ; 0, p, 0 ; 0,0, p)$, where $p$ is the common value of $p_{x x}, p_{y y}, p_{z z}$. The number $p$ is called the pressure of the perfect fluid and it is well to remember that the concept of pressure, as here defined, does not exist for a non-perfect or viscous fluid. For a perfect fluid the equations of motion are

$$
\rho \frac{d u}{d t}=\rho X-\frac{\partial p}{\partial x}, \rho \frac{d v}{d t}=\rho Y-\frac{\partial p}{\partial y}, \rho \frac{d w}{d t}=\rho Z-\frac{\partial p}{\partial z},
$$

to which must be added the boundary conditions

$$
F+l p=0, G+m p=0, H+n p=0,
$$

where $p$ denotes, in these latter equations, the pressure at the boundary of the fluid. It is clear from (16) that an acceleration potential $Q$ exists for a perfect fluid if (1) $(X, Y, Z)$ are derivable from a potential function $V$, that is, if

$$
X=-\frac{\partial V}{\partial x}, \quad Y=-\frac{\partial V}{\partial y}, \quad Z=-\frac{\partial V}{\partial z},
$$

and (2) if

$$
\frac{1}{\rho} \frac{\partial p}{\partial x}=\frac{\partial P}{\partial x}, \frac{1}{\rho} \frac{\partial p}{\partial y}=\frac{\partial P}{\partial y}, \quad \frac{1}{\rho} \frac{\partial p}{\partial z}=\frac{\partial P}{\partial z},
$$

where $P$ is any function of $x, y, z$ and $t$. Then $Q=V+P$. We shall be concerned mainly with the case where the fluid is homogeneous, that is, $\partial \rho / \partial x=0, \partial \rho / \partial y=0, \partial \rho / \partial z=0$, and in this case $P=p / \rho$ so that

$$
Q=V+\frac{p}{\rho}
$$


If our first condition is satisfied but not our second we have from (9)

$$
\begin{aligned}
& \frac{d \Gamma}{d t}=\int\left[\frac{\partial\left(p, \frac{1}{\rho}\right)}{\partial(y, z)} \delta(y, z)+\right. \frac{\partial\left(p, \frac{1}{\rho}\right)}{\partial(z, x)} \delta(z, x) \\
&\left.\quad+\frac{\partial\left(p, \frac{1}{\rho}\right)}{\partial(x, y)} \delta(x, y)\right] \\
&=\int \delta\left(p, \frac{1}{\rho}\right)=\oint_{C} p \delta\left(\frac{1}{\rho}\right) .
\end{aligned}
$$

5. Bernoulli's Theorem connecting Pressure and Velocity. When an acceleration potential $Q$ exists the three equations $d u / d t=-\partial Q / \partial x$, etc., may be written in the form

$$
\frac{\partial u}{\partial t}+\eta w-\zeta v=-\frac{\partial}{\partial x}\left(Q+\frac{1}{2} q^{2}\right),
$$

and so forth, where $(\xi, \eta, \zeta)$ are the vorticity components and $q^{2}=u^{2}+v^{2}+w^{2}$ is the square of the velocity. These yield at once the equation

$$
\frac{d}{d t}\left(Q+\frac{1}{2} q^{2}\right)=\frac{\partial Q}{\partial t},
$$

so that when $Q$ is free of $t, Q+q^{2} / 2$ is a first integral of the equations (3). In other words $Q+q^{2} / 2$ has a constant value along any one path but it will, in general, vary from path to path. If, however, the motion is steady $(\partial u / \partial t=0$, etc.), and irrotational $\left(\xi=0\right.$, etc.), we have $\partial\left(Q+q^{2} / 2\right) / \partial x=0$, etc., so that $Q+q^{2} / 2$, being free of $t$, is a number independent of $x$, $y, z$ and $t$. In the application with which we shall be concerned we shall have $V=0, P=p / \rho$, so that $Q=V+P=p / \rho$, with $\rho$ a numerical constant. Hence we may write

$$
p+\frac{1}{2} \rho q^{2}=p_{0} ; \text { or } p=p_{0}-\frac{1}{2} \rho q^{2},
$$

where $p_{0}$ is a constant throughout the fluid. Since $p_{0}$ is 
what $p$ becomes wherever $q=0$, it is called the static pressure. The additional term $-\rho q^{2} / 2$ is called the dynamic pressure.

6. The Forces and Turning Moments Experienced by Solids in the Fluid. The equations (16) may be written in the form

$$
\rho X-\frac{\partial p}{\partial x}=\rho \frac{d u}{d t}=\rho \frac{\partial u}{\partial t}+\frac{\partial}{\partial x}\left(\rho u^{2}\right)+\frac{\partial}{\partial y}(\rho u v)+\frac{\partial}{\partial z}(\rho u w),
$$

and so forth, on using (5). Upon integrating this equation throughout the region of the fluid between the immersed solid, or solids, $S$ and any convenient outer boundary surface $S^{\prime}$, and using the fact that at any point of the surface of a solid the velocity of the fluid is tangential to the surface, we find that the force components $\left(F_{x}, F_{y}, F_{z}\right)$ exerted by the fluid on the immersed solids are given by equations of the form

$$
\text { (22) } \begin{aligned}
F_{x}=\int \rho X d \tau & -\int \frac{\partial}{\partial t}(\rho u) d \tau \\
& -\int \rho u(l u+m v+n w) d S^{\prime}-\int p l d S^{\prime},
\end{aligned}
$$

where $(l, m, n)$ are the direction cosines of the normal to $d S^{\prime}$, drawn away from the fluid. In the cases to which we shall apply this result we shall assume the applied mass forces negligible $(X=0$, etc.), the motion steady $(\partial u / \partial t=0$, etc.) and irrotational (so that (20) is applicable) and the fluid is compressible and homogeneous; we find then

$$
\text { (23) } \begin{aligned}
F_{x} & =\frac{\rho}{2} \int\left\{l\left(u^{2}+v^{2}+w^{2}\right)-2 u(l u+m v+n w)\right\} d S^{\prime} ; \\
F_{y} & =\quad ; \quad F_{z}=.
\end{aligned}
$$

In a similar way, the moments $\left(M_{x}, M_{y}, M_{z}\right)$ about the coordinate axes of the forces exerted by the fluid on the immersed obstacle are given by equations of the form

$$
\begin{aligned}
M_{x}=\frac{\rho}{2} & \int\left\{(y n-z m)\left(u^{2}+v^{2}+w^{2}\right)\right. \\
& -2(y w-z v)(l u+m v+n w)\} d S^{\prime},
\end{aligned}
$$


and so forth. It is with these forces and moments that we shall be mainly concerned and the significance of the formulas given lies in the fact that the desired values are obtained by integration over an arbitrary surface $S^{\prime}$ enclosing the obstacles. It will be usually convenient, when the fluid is supposed infinite in extent, to regard $S^{\prime}$ as receding to infinity and we see that a knowledge of the nature of the flow at infinity suffices to determine the forces exerted on the solids by the fluid and also the moments of these forces.

\section{B. Two-Dimensional Irrotational Motion}

7. The Complex Velocity and Potential. In many applications we may assume, at any rate to a first approximation, that the velocity of each particle is parallel to a given plane and independent of its distance from it. Taking the given plane as our $(x, y)$ plane we have $w=0$ and $(y, v)$ independent of $z$. We shall find it convenient to introduce the complex variable $x+i y$ and as we shall not have occasion to use the symbol $z$ in this section in its previous sense we shall denote $x+i y$ by $z$. Assuming the fluid to be incompressible we have, from (6), the relation

$$
\frac{\partial u}{\partial x}+\frac{\partial v}{\partial y}=0
$$

and if we further assume the motion to be irrotational we have

$$
\zeta \equiv \frac{\partial v}{\partial x}-\frac{\partial u}{\partial y}=0
$$

The equations (25) and (26) are equivalent to the statement that $u-i v$ is a function of $z$. We shall denote this function by $w(z)$, since there is no possibility of confusion with the third velocity component which is now everywhere zero; $w(z)$ is called the complex velocity. Upon introducing the function $f(z)$ defined, save for an additive constant, by the equation 


$$
\frac{d f}{d z}=w(z)
$$

and denoting the real and imaginary parts of $f$ by $\phi$ and $\psi$, respectively, we have, from (27),

$$
u=\frac{\partial \phi}{\partial x}=\frac{\partial \psi}{\partial y}, \quad v=\frac{\partial \phi}{\partial y}=-\frac{\partial \psi}{\partial x} .
$$

Hence $\phi$ is the velocity potential and the level curves $\psi=$ constant are the stream-lines. The function $f$ is called the complex potential. The equations (28) tell us that the resolved part $v_{s}$ of the velocity vector in any direction $s$ is given by

$$
v_{s}=\frac{\partial \phi}{\partial s}=\frac{\partial \psi}{\partial\left(s+\frac{\pi}{2}\right)} .
$$

Once the complex potential $f$ is known the velocity components $(u, v)$ follow upon differentiation and from these the pressure at any point may be calculated by means of (20), the motion being assumed to be steady.

8. The Flow around a Circular Cylinder. We shall suppose that the cylinder, of radius $a$ and with generators perpendicular to the $(x, y)$ plane, has a uniform velocity of translation $V$ making an angle $-\alpha$ with the positive $x$ axis. The flow relative to the cylinder will, therefore, have a velocity $V$ making an angle $\pi-\alpha$ with the positive $x$ axis at points remote from the cylindrical obstacle. In other words the complex velocity $w$ will have the value $V e^{i(\pi+\alpha)}=-V e^{i \alpha}$ at $z=\infty$ so that, at $z=\infty, f$ will have the form $-V e^{i \alpha} z$ + terms whose derivatives vanish at $z=\infty$. Since the circle in which the cylinder intersects the $(x, y)$ plane must be a portion of a stream line, $\psi$ must be constant along it and this constant may be taken to be zero since $f$ is undefined to the extent of an additive constant; hence the correspondence set up between points in the $z$ plane and points of the 
$f=\phi+i \psi$ plane is such that points on the circle in the $z$ plane correspond to points on the axis of reals in the $f$ plane. If the center of the circle in the $z$ plane be denoted by $z_{0}$ and if $z_{0} \pm a e^{i \delta}$ are two diametrically opposite points on the circle the relation

$$
\frac{f-f_{0}}{f+f_{0}}=C\left(\frac{z-z_{0}-a e^{i \delta}}{z-z_{0}+a e^{i \delta}}\right)^{2}
$$

will set up a correspondence of the kind desired, the ends of the segment of the real axis in the $f$ plane being denoted by $\pm f_{0}$ and $C$ being a constant. In order that $f$ may have the proper form, $f=-V e^{i \alpha} z+\cdots$, at $\infty$, it is necessary that $C=1$ and $\delta=\pi-\alpha, f_{0}=2 a V$. The terms in $f$ whose derivatives vanish at $z=\infty$ may include, since $f$ need not be uniform owing to the fact that the portion of the $z$ plane outside the circle is not simply connected, a term of the form $A \log \left(z-z_{0}\right)$; for if $A$ is a pure imaginary, the imaginary part of $A \log \left(z-z_{0}\right)$ is constant as we go along the circle in the $z$ plane. If we write $A=-i \Gamma /(2 \pi)$, the quantity $\Gamma$ will be the circulation around any closed curve in the $(x, y)$ plane which enlaces the cylinder once and we have

$$
f=-V\left[\left(z-z_{0}\right) e^{i \alpha}+\frac{a^{2} e^{-i \alpha}}{z-z_{0}}\right]-\frac{i \Gamma}{2 \pi} \log \left(z-z_{0}\right)
$$

We shall see in the next paragraph how $\Gamma$ is determined for obstacles with a sharp trailing edge. It was precisely this determination of $\Gamma$ which constituted the first significant result belonging to what we have termed the modern period.

On differentiating $f$ it is seen that there are two points where $w$ is zero. If $\Gamma \leqq 4 \pi a V$, these two points are on the circle and are symmetrically situated with respect to the diameter of the circle which is perpendicular to the direction of flight. The two points are known as rest points and they are distinguished from one another by the terms front and rear. If the argument of the rear rest-point be denoted by $\pi+\beta$ we have 


$$
\Gamma=4 \pi a V \sin (\alpha+\beta)
$$

9. Flow around an Airfoil. If we have an obstacle in the $z$ plane which can be mapped on a circle in an auxiliary $\zeta$ plane by means of a relationship of the type

$$
\zeta=z+\frac{a_{1}}{z}+\frac{a_{2}}{z^{2}}+\cdots
$$

which makes the points at $\infty$ in the two planes correspond to one another, it is clear from (30) that the desired complex potential is given by

$$
f=-V\left[\left(\zeta-\zeta_{0}\right) e^{i \alpha}+\frac{a^{2} e^{-i \alpha}}{\zeta-\zeta_{0}}\right]-\frac{i \Gamma}{2 \pi} \log \left(\zeta-\zeta_{0}\right)
$$

where $\zeta$ is defined by (32), $a$ is the radius, and $\zeta_{0}$ the center of the circle in the auxiliary plane. We shall be interested mainly in obstacles whose contour is of the type illustrated in Fig. 1; these have a cusp or sharp double point at the trailing edge $T$ and so $d z / d \zeta$ will be zero there. Now the complex velocity

$$
w=\frac{d f}{d z}=\frac{d f}{d \zeta}: \frac{d z}{d \zeta}
$$

and this will be infinite at the trailing edge unless $d f / d \zeta$ is zero there. This condition suffices to determine the unknown circulation $\Gamma$. Physically it amounts to saying that for a stream-lined airfoil the flow is smooth at the trailing edge. Geometrically stated, the rear rest-point on the circle in the auxiliary $\zeta$ plane must be mapped on the trailing edge of the obstacle in the $z$ plane.

10. The Blasius Formulas for Lift and Turning-Moment. If $d s^{\prime}$ denote the element of arc of any curve $C^{\prime}$ enclosing the contour of the obstacle, the components of the force per unit length exerted on the obstacle by the fluid are, from (23), 


$$
\begin{aligned}
& F_{x}=\frac{\rho}{2} \oint_{C^{\prime}}\left\{l\left(u^{2}+v^{2}\right)-2 u(l u+m v)\right\} d s^{\prime}, \\
& F_{y}=\frac{\rho}{2} \oint_{C^{\prime}}\left\{m\left(u^{2}+v^{2}\right)-2 v(l u+m v)\right\} d s^{\prime},
\end{aligned}
$$

where $(l, m)$ are the $(x, y)$ direction cosines of the outward drawn normal to $C^{\prime}$. Since $l=d y^{\prime} / d s^{\prime}$ and $m=-d x^{\prime} / d s^{\prime}$, we readily find that

$$
\bar{F} \equiv F_{x}-i F_{y}=\frac{i \rho}{2} \oint_{C^{\prime}} w^{2} d z
$$

This important formula, due to Blasius, enables us to write down at once $F_{x}$ and $F_{y}$ when the development of $w^{2}$ near $z=\infty$ is known.

Similarly the turning moment $M$, about an axis through the origin perpendicular to the $(x, y)$ plane, of the forces exerted by the fluid on a unit length of the cylinder, being equal to

$$
\frac{\rho}{2} \oint_{C^{\prime}}\left\{(x m-y l)\left(u^{2}+v^{2}\right)-2(x v-y u)(l u+m v)\right\} d s^{\prime},
$$

is seen to be the real part of

$$
-\frac{\rho}{2} \oint_{C^{\prime}} z w^{2} d z
$$

that is,

$$
M=\mathrm{R} \cdot \mathrm{P} \cdot\left(-\frac{\rho}{2} \oint_{C^{\prime}} z w^{2} d z\right)
$$

In order to apply these results we turn to (32) and (33). Thus, from

$$
\zeta=z\left(1+\frac{a_{1}}{z^{2}}+\cdots\right)
$$

we find 


$$
\frac{1}{\zeta}=\frac{1}{z}-\frac{a_{1}}{z^{3}}+\cdots ; \frac{1}{\zeta^{2}}=\frac{1}{z^{2}}-\frac{2 a_{1}}{z^{4}}+\cdots,
$$

whence

$$
\frac{d f}{d \zeta}=-V e^{i \alpha}-\frac{i \Gamma}{2 \pi z}+\left(V a^{2} e^{-i \alpha}-\frac{i \Gamma \zeta_{0}}{2 \pi}\right) \frac{1}{z^{2}}+\cdots .
$$

Multipliying this by

we find

$$
\frac{d \zeta}{d z}=1-\frac{a_{1}}{z^{2}}+\cdots
$$

$$
\begin{aligned}
w=-V e^{i \alpha} & -\frac{i \Gamma}{2 \pi z}+\left(V a^{2} e^{-i \alpha}-\frac{i \Gamma \zeta_{0}}{2 \pi}+a_{1} V e^{i \alpha}\right) \frac{1}{z^{2}}+\cdots \\
w^{2}=V^{2} e^{2 i \alpha} & +\frac{i V \Gamma e^{i \alpha}}{\pi z} \\
& -\left(\frac{\Gamma^{2}}{4 \pi^{2}}+2 V^{2} a^{2}-\frac{i \Gamma \zeta_{0} V e^{i \alpha}}{\pi}+2 a_{1} V^{2} e^{2 i \alpha}\right) \frac{1}{z^{2}}+\cdots
\end{aligned}
$$

From this expression it follows, by (34), that

$$
\begin{aligned}
& \bar{F} \equiv F_{x}-i F_{y}=-i \rho V \Gamma e^{i \alpha} \\
& F \equiv F_{x}+i F_{y}=i \rho V \Gamma e^{-i \alpha} .
\end{aligned}
$$

In other words the force per unit length exerted by the fluid on the cylindrical obstacle has the magnitude $\pm \rho V \Gamma$ and is perpendicular to the direction of flight, the sense of rotation from the direction of flight to the direction of the force being that of the circulation $\Gamma$. See Fig. 2. This force per unit length is called the lift per unit length and is denoted by $L$, so that we have the Kutta-Joukowski formula

$$
L=\rho V \Gamma \text {. }
$$

If the potential function $f$ were assumed uniform, $\Gamma$ would be zero and there would be no force experienced by the obstacle; a result known as d'Alembert's paradox. The component of the force in the direction opposite to the direction of flight is called the drag and the drag per unit length is de- 
noted by $D$. We see, then, that the present theory gives $D=0$, which is not in accord with experience. It will appear in the next section that when account is taken of the fact that the length of the obstacle is not infinite this discrepancy can be removed.

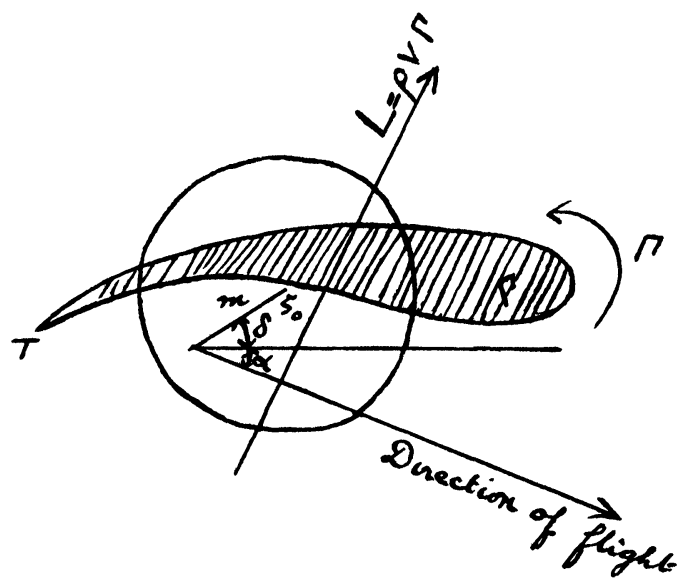

FIG. 2

If we introduce the notation $\zeta_{0}=m e^{i \delta}, a_{1}=-b^{2} e^{2 i \gamma}$, where $m, \delta, b$ and $\gamma$ are all real, it follows that

$$
M=\rho V \Gamma m \cos (\alpha+\delta)+2 \pi \rho b^{2} V^{2} \sin 2(\alpha+\gamma),
$$

and hence the turning moment about the center $\zeta_{0}=0$ of the circle in the auxiliary $\zeta$ plane, the two planes being supposed superposed, is

$$
M_{0}=2 \pi \rho b^{2} V^{2} \sin 2(\alpha+\gamma) .
$$

11. The Circular Arc and Straight Line Profile. The relationship $((\zeta-l) /(\zeta+l))^{2}=A(z-c / 2) /(z+c / 2)$, where $A$ is any constant, maps a doubly covered circular arc through the points $\pm c / 2$ in the $z$ plane on a circle through the points $\pm l$ in the $\zeta$ plane. In order that the mapping may be of the type (32) we must have $A=1, l=c / 4$ and the mapping is

$$
z=\zeta+\frac{c^{2}}{16 \zeta}, \quad \zeta=z-\frac{c^{2}}{16 z}+\cdots,
$$


it being necessary to take that one of the two determinations of $\zeta$ which tends to $\infty$ with $z$. If we denote by $2 \epsilon$ the angle of the circular arc, we have

(40) $\zeta_{0}=i l \operatorname{ctn} \epsilon \cdot=\frac{i c}{4} \operatorname{ctn} \epsilon, \quad a=l \csc \epsilon=\frac{c}{4} \csc \epsilon$

$$
\beta=\frac{\pi}{2}-\epsilon, \quad b^{2}=\frac{c^{2}}{16}, \quad \gamma=0,
$$

so that, by (31)

$$
\Gamma=\pi c V \csc \epsilon \cos (\alpha-\epsilon)
$$

(42) $L=\pi \rho V^{2} c \csc \epsilon \cos (\alpha-\epsilon), \quad M_{0}=\left(\pi \rho c^{2} V^{2} \sin 2 \alpha\right) / 8$.

The distance $h$ of the lifting force $L=\rho V \Gamma$ from the center of the auxiliary circle is

$$
h=M_{0} / L=c \sin 2 \alpha \sin \epsilon /[8 \cos (\alpha-\epsilon)] .
$$

The particular case where $\epsilon=\pi / 2$ is very important. Here we are dealing with a plane wing flying at an angle of attack $\alpha$ and we find

$$
\Gamma=\pi c V \sin \alpha, \quad L=\pi c \rho V^{2} \sin \alpha, \quad h=c \cos \alpha / 4 .
$$

The number $c$ is the length of the chord of the wing and the expression for $h$ indicates that the point where the line of action of $L$ meets the chord, that is, the center of pressure, does not vary with $\alpha$ but remains constantly one-quarter of the length of the chord from the leading edge; for $\zeta_{0}$ is now the middle point of the chord. The number $c_{L}=L /\left(\frac{1}{2} \rho V^{2} c\right)$ is called the lift coefficient $c_{L}$ and we have the result that for a plane wing

$$
c_{L}=2 \pi \sin \alpha
$$

For small angles of attack we may put

$$
c_{L}=2 \pi \alpha \text {. }
$$


so that there is a linear relation connecting $c_{L}$ and $\alpha$. This prediction is verified by experience for angles of attack up to about $15^{\circ}$; but the factor of proportionality $2 \pi$ is found to be about $40 \%$ too large. The reason for this discrepancy is that the wing is not infinite in length and a corrected expression will be given in the next section.

12. Joukowsky Profiles and their Extensions. The relationship between $z$ and $\zeta$ which is expressed by (39) maps the exterior of a circle through the points $\pm c / 4$ in the $\zeta$ plane upon the exterior of a doubly covered circular arc, with ends at the points $\pm c / 2$, in the $z$ plane. A profile suitable for airfoils is obtained if the same mapping relationship is applied to a circle through the point $-c / 4$ but not through the point $+c / 4$ in the $\zeta$ plane. If the circle does not differ very much from the circle through the points $\pm c / 4$ which maps into the doubly covered circular arc, the contour will have a rounded leading edge and a sharp trailing edge. A linear fractional substitution on a complex variable being resolvable into a translation, rotation, reflexion in a line and an inversion may be called a generalized inversion and it is evident that the mapping (39) may be written as

$$
z^{\prime}=\zeta^{\prime 2}, \quad z^{\prime}=\frac{z-\frac{c}{2}}{z+\frac{c}{2}}, \quad \zeta^{\prime}=\frac{\zeta-\frac{c}{4}}{\zeta+\frac{c}{4}} .
$$

Now a generalized inversion sends circles into circles (straight lines being regarded as circles through the point at infinity). The generalized inversion in the $\zeta$ plane, written down in (46), sends any circle through $-c / 4$ in this plane into a straight line and this is sent by the square-mapping $z^{\prime}=\zeta^{\prime 2}$ into a parabola in the $z^{\prime}$ plane. The profile obtained is then the generalized inverse of a parabola. Such profiles are known as Joukowsky profiles. Generalizations are obtained by writing, instead of (46), 


$$
z^{\prime}=\zeta^{\prime n}, \quad z^{\prime}=\frac{z-\frac{c}{n}}{z+\frac{c}{n}}, \quad \zeta^{\prime}=\frac{\zeta-\frac{c}{2 n}}{\zeta+\frac{c}{2 n}}
$$

which is of the form (32) near $z=\infty$. This sends the exterior of a circle through the points $\pm c /(2 n)$ in the $\zeta$ plane into the exterior of a region bounded by two circular arcs through the points $\pm c / n$ in the $z$ plane, the angle between the two arcs being $(2-n) \pi$. By taking a circle in the $\zeta$ plane through $-c /(2 n)$ but not exactly through $+c /(2 n)$ we obtain a profile with a rounded leading edge and a sharp trailing edge; it has the crescent formed by the two circular arcs as a "skeleton" just as the Joukowsky profile has the doubly covered circular arc as skeleton. The essential difference is that the trailing edge is now a double point of the contour and not a cusp as in the case of a Joukowsky profile. The new profiles are obtained by applying a generalized inversion to the curves obtained by applying the $n$th power mapping $z^{\prime}=\zeta^{\prime n}$ to a straight line (passing nearly through the origin) in the $\zeta^{\prime}$ plane.

\section{The Induced Drag of a Finite Airfoil}

13. The Formulas for the Lift and Drag. It has been seen in the previous section that when the length of the airfoil is assumed infinite it experiences, owing to the circulation around it, a force at right angles to the direction of flight; in other words there is a lift but no drag. In order to give an explanation of this discrepancy between theory and experiment Prandtl advanced the idea that since an actual airfoil is not infinite in length the circulation around it may vary from point to point along its length. Under this hypothesis the flow loses at once its two-dimensional character but it is assumed that the results obtained for the infinite wing may be used as first approximations; the fact that the circulation is not constant along the span of the airfoil introduces a correction to this approximation. To get an 
estimate of the nature and amount of this correction we shall consider the case of a plane wing whose chord $c$ is small in comparison with its length or span $s$ and we shall suppose the airfoil sufficiently represented by its trailing edge (it is then called a lifting line). If we introduce a coordinate system with its origin at the middle of the lifting line (see Fig. 3), we may draw the $x$ axis in the direction of flight, the $z$ axis vertically downwards and the $y$ axis along the lifting line. The circulation around the wing in any plane perpendicular to it is assumed to be a function $\Gamma(y)$

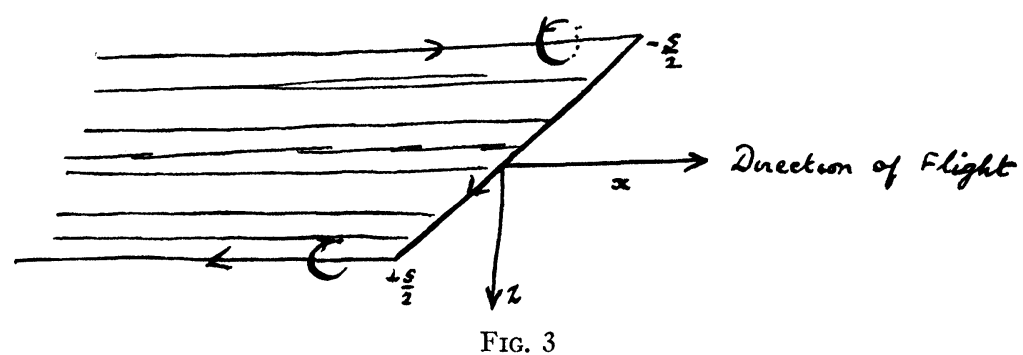

of $y$. If then we consider the ring-shaped area bounded by two closed curves enlacing the wing, one in the plane $y$ and the other in the plane $y+\Delta y$, there will be a flux of vorticity through this area, outwards from the wing, of amount $-(d \Gamma / d y) \Delta y$. (See (8).) We assume that this flux is concentrated at the trailing edge and is in the direction of the negative $x$ axis, that is, is in the direction opposite to the direction of flight. Hence there will be a sheet of vortices trailing off from the trailing edge of the wing such that the strength of the vortices leaving an element $\Delta y$ is $-(d \Gamma / d y) \Delta y$. Now if we have a single rectilinear vortex filament of strength $\mu$ in an indefinitely extended fluid which is at rest at infinity, the velocity distribution must be symmetrical around it and hence at a distance $r$ from it the velocity must be of magnitude $\mu /(2 \pi r)$. We assume that for a semi-infinite filament extending, as in the present case, along the negative $x$ axis, the magnitude of the velocity at a point along the $y$ axis distant $r$ from the filament will be $\mu /(4 \pi r)$. The direction 
of the velocity will be that of the positive or negative $z$ axis. This argument leads to the conclusion that the presence of the semi-infinite strip of trailing vortices induces at any point $y$ of the lifting line a velocity $w(y)$ in the direction of the $z$ axis, that is downwards, of amount

$$
w(y)=\frac{1}{4 \pi} \int_{-s / 2}^{s / 2} \frac{(d \Gamma / d y)_{y=y^{\prime}}}{y-y^{\prime}} d y^{\prime} .
$$

This assumption of an induced downwash is the cardinal one of Prandtl's theory for the finite wing. For an infinite plane wing flying at a small angle of attack $\alpha$ with uniform velocity $V$ an easy evaluation of the indeterminate form $d f / d z$ shows that the relative velocity of the wind at the trailing edge has the magnitude $V \cos \alpha$ and the direction of the negative chord of the wing and for small angles of attack $\alpha$ this can be regarded as of magnitude $V$ in the direction opposite to the direction of flight. The net effect of comDounding with this relative velocity $V$ the downwash $w$ is to rotate the relative velocity through the angle $\tan ^{-1}(w / V)$ and since $w$ is merely a small corrective term this can be equated to $w / V$. This rotation of the relative wind, due to the sheet of trailing vortices, may be regarded as equivalent to a rotation of the direction of flight through the same angle, that is to a diminution of the angle of attack (which is the angle from the direction of flight to the chord of the wing). The diminished angle $\alpha_{e}$ is called the effective angle of attack (as opposed to the geometric angle of attack $\alpha_{\theta}$ ) and we have the equation

$$
\alpha_{e}=\alpha_{\vartheta}-\frac{w}{V} .
$$

On making the assumption that we can apply the results developed for the infinite wing, once we have replaced the geometric angle of attack by the effective angle of attack, we find that the lift per unit length $L^{\prime}$ at any point $y$ on the span of the wing is given by 


$$
2 \pi c \cdot \frac{1}{2} \rho V^{2} \cdot\left(\alpha_{o}-\frac{w}{V}\right),
$$

and on using $L^{\prime}=\rho V \Gamma$ and equation (48), we have the equation

$$
\Gamma(y)=\pi c V \alpha_{g}-\frac{c}{4} \int_{-s / 2}^{s / 2} \frac{(d \Gamma / d y)_{y=y^{\prime}}}{y-y^{\prime}} d y^{\prime}
$$

for the unknown function $\Gamma(y)$. For a given airfoil both $c$ and $\alpha_{g}$ will be given functions of $y$; if $\alpha_{g}$ is not constant the airfoil is said to be twisted. It is apparent from (49) that even when $\alpha_{g}$ is constant $\alpha_{e}$ may vary owing to the possibility of a variable downwash $w(y)$. This variability of $\alpha_{e}$ is known as aerodynamic twisting of the airfoil.

Since the lift experienced by an infinite wing is perpendicular to the direction of flight and since the effect of the downwash is to decrease, in effect, the angle of attack by $w / V$, the direction of the force at any point of the wing will be rotated through an angle $w / V$ from the direction of lift. It is this rotation of the direction of the force which introduces a component in the direction opposite to that of flight. Once $\Gamma(y)$ is determined from (50), the total lift $L$ and $\operatorname{drag} D$ experienced by the wing are given by the expressions

$$
L=\rho V \int_{-s / 2}^{s / 2} \Gamma d y, \quad D=\rho \int_{-s / 2}^{s / 2} w \Gamma d y,
$$

(where we have identified the sine of $w / V$ with $w / V$ ). The drag $D$ given by (51) is known as induced drag, it being a matter of experience that it is too small by a certain amount depending on the shape of the airfoil and known as the profile drag.

14. The Solution of the Integral Equation and the Determination of the Airfoil of Minimum Induced Drag having a Given Lift. In order to obtain a numerical solution of $(50)$ we make the substitution $y=-(s / 2) \cos \theta$, so that $\theta$ runs from 0 to $\pi$ along the span of the wing and imagine $\Gamma(y)=F(\theta)$ developed 
in a Fourier sine series (in order that there may be no discontinuity in $\Gamma(y)$ at the wing tips, $y= \pm s / 2$, we must have $\Gamma( \pm y / 2)=0)$. Writing this series as $A_{1} \sin \theta+A_{2} \sin 2 \theta$ $+\cdots$, we see from the fact that $\Gamma(y)$ is symmetric about the middle of the wing that $A_{2}=A_{4}=A_{6}=\cdots=0$. In actual calculations it will be sufficient to consider the four terms $A_{1} \sin \theta+A_{3} \sin 3 \theta+A_{5} \sin 5 \theta+A_{7} \sin 7 \theta$ in the development of $\Gamma(y)$. A simple substitution in (50) and a knowledge of the fact that

$$
\int_{0}^{\pi} \frac{\cos n \phi d \phi}{\cos \phi-\cos \theta}=\pi \frac{\sin n \theta}{\sin \theta}
$$

gives the equation

$$
\sum_{1}^{\infty} A_{n} \sin n \theta\left\{\sin \theta+n \frac{\pi c}{2 s}\right\}=\pi c V \alpha_{0} \sin \theta
$$

for the determination of the coefficients $A_{n}$. On substituting the values $\theta=\pi / 8, \theta=\pi / 4, \theta=3 \pi / 8, \theta=\pi / 2$, in this and dropping the terms after $A_{7}$ we have four linear equations for the determination of the four coefficients $A_{1}, A_{3}, A_{5}$, and $A_{7}$.

The coefficients $A_{n}$ being supposed known, the expressions for the lift $L$ and the $\operatorname{drag} D$ in terms of them follow readily from the formulas (51). We find

$$
L=\rho V s A_{1} / 4, \quad D=\frac{\pi \rho}{8} \sum_{1}^{\infty} n A_{n}^{2},
$$

so that the lift depends only on the first term in the Fourier development of $\Gamma(y) \equiv F(\theta)$. The downwash $w$ is given by

$$
w(y)=\frac{1}{2 s} \sum_{1}^{\infty} \frac{n A_{n} \sin n \theta}{\sin \theta},
$$

and the drag $D$ by

$$
D=\frac{\pi \rho}{8} \sum_{1}^{\infty} n A_{n}^{2}
$$

It follows that $D$ is a minimum, for a given value of $L$, when 
the remaining coefficients $A_{3}, A_{5}, \cdots$ after $A_{1}$ in the Fourier development of $F(\theta)$, are zero. In this case the downwash has the constant value $A_{1} /(2 s)$ and there is no aerodynamic twisting of the wing. Since

$$
\Gamma(y)=A_{1} \sin \theta=A_{1}\left(1-\frac{4 y^{2}}{s^{2}}\right)^{1 / 2}
$$

the graph of $\Gamma(y)$ against $y$ is an ellipse and the wing is said to be elliptically loaded. Since for an infinite plane wing the lift per unit length is, for a given angle of attack, proportional to the chord $c$ (see (43)), we may surmise that an elliptically loaded wing would be approximated to by one whose profile is approximately elliptical. A criterion of good design of an airfoil is that, for a given $L, D$ should be as small as possible and so for a well designed airfoil we may assume the loading approximately elliptical.

15. Comparison with Experiment. For a finite wing hav-. ing a constant chord $c$ the ratio of the span $s$ to the chord $c$ is known as the aspect ratio. For a plane wing whose chord is not constant we first define the mean chord $c$ as the quotient of the area $S$ of the wing by its span $s$; then the aspect ratio $A$ is the ratio of the span of the wing to its mean chord. Hence $A=s^{2} / S$. The lift coefficient $c_{L}$ of the wing is defined by the equation

$$
c_{L}=L /\left(\frac{1}{2} \rho V^{2} S\right),
$$

and there is a similar equation defining the drag coefficient. For an elliptically loaded wing we have, by (53) and (56),

$$
c_{L}=\frac{\pi A}{2 V s} A_{1},
$$

while the downwash $w$ has the constant value

$$
w=\frac{A_{1}}{2 s}=\frac{V}{\pi A} c_{L} .
$$

Hence the diminution in the angle of attack, induced by the 
trailing vortices, is $c_{L} /(\pi A)$. The total lift $L$ of the wing is

$$
2 \pi \alpha_{e} \cdot \frac{1}{2} \rho V^{2} \cdot \int_{-8 / 2}^{s / 2} c d y=2 \pi \alpha_{e} \cdot \frac{1}{2} \rho V^{2} \cdot S,
$$

so that $c_{L}=2 \pi \alpha_{e}$ and we have

$$
\alpha_{g}=\alpha_{e}+\frac{c_{L}}{\pi A}=\alpha_{e}\left(1+\frac{2}{A}\right) .
$$

The aspect ratio of a wing is usually about 6 , so that the lift formula for the infinite wing (using $\alpha_{g}$ ) might be expected to give a result which is too large in the ratio $4: 3$. This is confirmed by experience.

The drag coefficient $c_{D}$ has, for an elliptically loaded wing, the value $\pi \rho A_{1}^{2} / 8$ (by (55)) so that, by (57),

$$
c_{D}=c_{L}^{2} /(\pi A) \text {. }
$$

Hence a curve plotting $D$ against $L$ should be parabolic and this is verified experimentally; the actual curve obtained empirically indicates a relationship of the type

$$
c_{D}=c_{D_{0}}+c_{L}^{2} /(\pi A)
$$

and $c_{D_{0}}$ is called the coefficient of profile drag. The formulas (59) and (61) are of importance because they enable us to calculate the effect of different aspect ratios; thus for two airfoils having the same lift coefficient

$$
\alpha_{0}{ }^{\prime}-\alpha_{0}=\frac{c_{L}}{\pi}\left(\frac{1}{A^{\prime}}-\frac{1}{A}\right), \quad c_{D}{ }^{\prime}-c_{D}=\frac{c_{L}{ }^{2}}{\pi}\left(\frac{1}{A^{\prime}}-\frac{1}{A}\right) .
$$

\section{Concluding Remarks}

In the present address, I have tried to present in an elementary way some of those fundamental principles of modern hydrodynamical theory, which have proved useful in aeronautics, in the hope of interesting some of our hearers and readers in these questions. It would be easy to point out many unsolved problems and indeed a casual reading of the previous sections will show that even in the most elementary 
problems assumptions have continually to be made the legitimacy of which is at least not proved. We append a bibliography which has been deliberately restricted to those works which we would recommend to a student wishing to orient himself in the subject and to reach research problems as soon as possible.

\section{TEXTBOOKS}

I. H. Lamb, Hydrodynamics. 5th ed. Cambridge University Press. The standard text on hydrodynamics.

II. H. Glauert, The Elements of Aerofoil and Airscrew Theory. Cambridge University Press. A fine and elementary introduction to the modern problems and methods.

III. P. Appell, Traitê de Mécanique rationnelle, vol. 3. Gauthier Villars.

IV. L. Bairstow, Applied Aerodynamics. Longmans-Green.

V. R. von Mises, Fluglehre. Julius Springer.

These two texts are less mathematical and more physical than the other texts and are particularly valuable as complementing them.

VI. Fuchs-Hopf, Aerodynamik. R. C. Schmidt. This is volume 2 of a Handbuch der Flugzeugkund, and is very complete.

VII. A. Betz, Tragflügel und Hydraulische Maschinen. Handbuch der Physik, vol. 7, pp. 215-288.

VIII. Th. v. Karman and T. Levi-Civita, Hydro- und Aerodynamik. J. Springer.

Many papers of interest will be found in the volumes of the Zeitschrift für Angewandte Mathematik and in the publications of the National Advisory Commitee for Aeronautics. Of these latter the following may be especially recommended:

IX. N.A.C.A. Report No. 116 (Prandtl). Government Printing Office, Washington, D.C.

X. N.A.C.A. Report No. 213 (Ames). Government Printing Office, Washington. D.C.

The Johns Hopkins University 